\title{
French Angiogenesis Society partners with Targeted Oncology
}

\author{
Fabrice Soncin
}

Received: 8 February 2010 /Accepted: 8 March 2010 /Published online: 25 March 2010

(C) Springer-Verlag 2010

Keywords Angiogenesis · Partnership

The French Angiogenesis Society (Société Française d'Angiogenèse) and the journal Targeted Oncology recently decided to become partners in their shared goals of promoting and disseminating clinical and fundamental cancer research. Angiogenesis corresponds to the formation of new blood vessels. It is critically important during embryonic development and throughout life, this process is also associated with several diseases. The theory that tumors depend on oxygen and blood supply was clearly and formally conceptualized in the 1970s. In the following years, the search for a "tumor angiogenic factor" and for endothelial-specific growth factors led to the discovery of vascular endothelial growth factor (VEGF) and many other angiogenic factors. We quickly learned that VEGF played a central role in angiogenesis and, more recently, VEGF was the first angiogenic factor against which a targeted therapy proved to be beneficial to cancer patients. This opened the novel path of so-called "anti-angiogenic" therapies, and several such therapies targeting VEGF and its receptors are now commonly used in the clinic; more will come, aimed at various other molecular and cellular agents of tumor angiogenesis.
The French Angiogenesis Society gathers clinicians and research scientists who show interest in the general field of angiogenesis. Although tumor angiogenesis is one of its major topics of interest, the Society is not strictly focused on cancer. Several members are working on other aspects of angiogenesis, such as embryonic development, retinal vessels, endothelial barriers, genetics, and gene regulation. Our next meeting will be held in southern France in May 2010, and we will be honored by presentations in English from several internationally recognized speakers. The readers of Targeted Oncology are most welcome to visit our website (www.angiogenese.fr) and, if interested, find their way to our next meetings.

The partnership with Targeted Oncology is clearly pertinent. In upcoming years, the Society will advertise its meetings and publish its reports in the journal. We sincerely hope that the readers of Targeted Oncology will find as much interest in our work as our members do in reading this journal. Should our partnership promote collaborations and the emergence of new, efficient therapies against cancer, both the journal and the Society would reach an important common goal.

F. Soncin $(\bowtie)$

Institut de Biologie de Lille,

Lille, France

e-mail: fabrice.soncin@ibl.fr 